\title{
SUBTYPE COASTLINE DETERMINATION IN URBAN COAST BASED ON MULTISCALE FEATURES: A CASE STUDY IN TIANJIN, CHINA
}

\author{
Yan Song*, 1 , Yeshuang Ai1, Haitian $\mathrm{Zhu}^{2,3}$ \\ ${ }^{1}$ China University of Geosciences, Wuhan, China - moonriver_song@163.com, 13007163487@163.com \\ ${ }^{2}$ National Satellite Ocean Application Service, Beijing 100081, China - n5717@163.com \\ ${ }^{3}$ Key Laboratory of Technology for Safeguarding of Maritime Rights and Interests and Application, State Oceanic Administration, \\ Guangzhou 510310;
}

KEY WORDS: Subtype Coastline, Multiscale Feature, Uncertainty, Remote Sensing, Tianjin

\begin{abstract}
:
In urban coast, coastline is a direct factor to reflect human activities. It is of crucial importance to the understanding of urban growth, resource development and ecological environment. Due to complexity and uncertainty in this type of coast, it is difficult to detect accurate coastline position and determine the subtypes of the coastline. In this paper, we present a multiscale feature-based subtype coastline determination (MFBSCD) method to extract coastline and determine the subtypes. In this method, uncertainty-considering coastline detection (UCCD) method is proposed to separate water and land for more accurate coastline position. The MFBSCD method can well integrate scale-invariant features of coastline in geometry and spatial structure to determine coastline in subtype scale, and can make subtypes verify with each other during processing to ensure the accuracy of final results. It was applied to Landsat Thematic Mapper (TM) and Operational Land Imager (OLI) images of Tianjin, China, and the accuracy of the extracted coastlines was assessed with the manually delineated coastline. The mean ME (misclassification error) and mean LM (Line Matching) are 0.0012 and $24.54 \mathrm{~m}$ respectively. The method provides an inexpensive and automated means of coastline mapping with subtype scale in coastal city sectors with intense human interference, which can be significant for coast resource management and evaluation of urban development.
\end{abstract}

\section{INTRODUCTION}

Coastline, as a kind of resource of coastal zone, contributes significantly to the resource development in coastal zones (Klemas et al., 2011). The changes of it in space and type can directly reflect erosion and silting-up, human exploitation and ecological environment (Vaidya et al., 2015). Automated analyses of the coastline, or coastline mapping techniques, are much important for coastal study and have been pursued (Gens et al., 2010).

Urban coast is a significant region to represent the interactions between human and marine (Xu et al., 2016). Recently, with the rapid developments of society and ecological environment, urban coasts have become widely distributed in coastal zones and attracted more attentions (Tamassoki et al., 2014). Due to complex human activities, this type of coast has the highly complex features in types and structures. Besides, the main subtypes have close relevance to the economic industry in this region, such as salt pans, aquaculture and ports. Therefore, in order to understanding the urbanization and human impacts in coastal city, effective methods of extracting the coastline in coastal city with subtype scale are desired (Dewi et al., 2016). Compared with conventional survey methods, remote sensing has more advantages for monitoring of coastline dynamics over a variety of spatial-temporal scales (Ghosh et al., 2015). Multispectral remote-sensing images, such as Landsat images with suitable spatial resolution and abundant spectral information, are still the major optical remote-sensing data source for coasts study (Wang et al., 2017; Du et al., 2014). In several recent studies, methods of coastline extraction from remote sensing imagery have been reviewed, in which the band or water index threshold methods are widely chosen (Dewan et al., 2008). Li, et al. (2016) applied the optimal threshold value to separate water and land by giving a cut-off value manually. The optimal threshold value for coastline extraction can be selected automatically or by man-machine interaction. This method is easy to implement and can obtain good accuracy for most coast types. Then, the edge detection approaches, such as Canny algorithm (Gu et al., 2016) and region growing (Zhan et al., 2017), are used for coastline extraction which is equal to an edge detection problem. Zhang, et al. (2013) proposed an automated region growing method, and successfully extract aquaculture coast. Classification methods which include supervised classification (Duru et al., 2017), unsupervised classification (Wang et al., 2017), soft classification (Muslim et al., 2006), and association rule method (Wang et al., 2010) are also recognized in some coastline extraction applications from multispectral Remote Sensing Images. Tatna, et al. (2016) presented an unsupervised fuzzy c-means classification (FCM) to observe the shoreline positions, and successfully accomplished the coastline extraction and change monitoring. The classification methods aim at classifying land and water, and coastline can be detection with the separation results of water and land. The approaches based on edge detection and classification can work well only when the edge features between the land and water can be clearly identified and distinguished. They have less robustness to image quality, even though soft or fuzzy classification can take uncertainty into account. It is complex and have no efficient during post processing. At the same time, in some applications, coastline also can be extracted with the fusion techniques which combine two or more data sources including optical data, SAR (Synthetic Aperture Radar) data, LIDAR (Light Detection And Ranging) data and digital orthoimage data (Yang et al., 2017). More data sources can provide more information. 
However, improving the performance of these methods for extraction of coastline in urban coast is still required. As known, coastline is the boundary of water and land. It is temporal and dynamic (Gens et al., 2010; Li et al., 2016). In the applications of coastline extraction, shoreline indicators including high water line (HWL), mean high water (MHW) and waterline are always used as a proxy to represent the 'true' coastline position [Gens et al., 2010; Liu et al., 2016]. Meanwhile, due to dynamic environment, complex land background, mixed pixel and image noises in coastal region, the uncertainty is induced ( $\mathrm{Li}$ et al., 2016). It is a huge factor to influence the accurate extraction of coastline. At present, the subtype of coastline is mainly decided by manual delineating (Pardo-Pascual et al., 2012). It is lack of automation and efficiency. As descripted above, the type and geometrical structure of coastline in urban coast are much complex. Besides, in the complex large scale scene, the effective features are easily submerged, and it is hard to obtain highlighting features for subtype coastline extraction and classification (Ding et al., 2014; Zhou et al., 2017).

To overcome these limitations, in this study, we present a method for coastline extraction and determine the subtypes of it, which is named multiscale feature-based subtype coastline determination (MFBSCD). It can effectively make the invariant features containing geometry and spatial structure stand out through the construction of Gaussian pyramid layer. With these features, the subtypes of coastline can efficiently be classified and determined. At the same time, in the procedure, an improved method called uncertainty-considering coastline detection (UCCD) is also proposed to separate water and land. It can obtain more accurate and reasonable coastline position with taking the uncertainty into consideration, which is the foundation of the subtype coastline classification and extraction. The effectiveness of MFBSCD are evaluated, and the results demonstrate that the method is appropriated for subtype coastline determination in urban coast.

\section{STUDY AREA AND DATA}

This study was carried out in Tianjin which is located in Northern China. There are port, saltpan and construction coastline in this region with total $300 \mathrm{~km}$ coastline. With the developments, the coastal area has been greatly urbanized. The coastline are constituted by the main subtypes (e.g., port coastline, saltpan coastline and construction coastline included), and each subtype has its own typical characteristic. The port coastline has high complexity in geometry. The saltpan coastline has apparent characteristic of water in the land area. The geometrical characteristic of construction coastlines is displayed by small curvature arcs, and there are buildings with impervious surfaces in the land area.

From 1986 to 2016, a total of 7 standard Level 1 Terraincorrected (L1T) products from Thematic Mapper (TM) and Operational Land Imager (OLI) of Landsat were obtained in 5 years intervals. All high quality images for the experiment were obtained in no sea ice period, which can eliminate the influence of sea ice during coastline extraction. With systematic geometric and atmospheric errors corrected, the surface reflectance was achieved through converting the at-sensor radiance (digital number, DN). After Gaussian filtering and masking with the vector boundary, the images for the experiment were produced.

\section{METHODOLOGY}

The overall method flow of the MFBSCD can be mainly divided into two parts: 1) the coastline position detection (Section 2.1), 2) the subtype coastline determination (Section 2.2).

\subsection{Coastline position detection with considering uncertainty}

The coastline position is the foundation for the coastline's study and application. As described above, coastline is the boundary of water and land, and is temporal and dynamic. With the computation of water index which can heighten the separability of water and land, the accurate coastline position is not easy detected because of uncertainty. So, in order to solve this problem, uncertainty-considering coastline detection (UCCD) method is proposed to separate water and land for more accurate coastline position, which can provide the critical foundation for the further process of the MFBSCD method.

Firstly, Automated Water Extraction Index (AWEI) of image, as a water index to provide a higher accuracy and the most stable threshold to classify pixels at the edge of water surface, is computed. The formulation of AWEI is expressed as:

$$
\mathrm{AWEI}=4 *\left(\rho_{\text {green }}-\rho_{\mathrm{SWIR} 1}\right)-\left(0.25 * \rho_{\mathrm{NIR}}+2.75 * \rho_{\mathrm{SWIR} 2}\right)
$$

where $\rho \mid \rho \in[0,1]$ represents the surface reflectance value of spectral bands, and AWEI value is decimal type and ranges from -3.0 to 4.0 .

After the normalization processing, the statistics histogram of normalized AWEI can be obtained and displayed in Figure 1a with taking the image in 1986 as an example. As shown, there are two peaks which respectively represent land (red line) and water (blue line). The distributions of them are approximately fitted with a double Gaussian function. Meanwhile, there some data values (green line) which are similarly equal to three standard deviations away from the mean and located in the area between two peaks of both distributions, and the frequency of each value is small. Through separating and extracting the pixels with these values, we can find that the objects these pixels represent mainly contain tidal flat, sediments with water, complex objects in land and image noises (Figure 1b). They are considered to be the mixture which is directly related to the uncertainty. As shown in Fig. 1b, there is no the mixture in the sea water region which is 100 percent contained in water part. Besides, the waterline, chosen as coastline indicator to represent coastline position, is the boundary of sea water and land. Therefore, it more reasonable to divide the mixture into land part, which can provide more accurate separated results of water-land.

In the segmentation of two classes, the between-class variance is widely chosen as energy function of the threshold method, such as typical OTSU method. The optimal threshold can be decided by maximizing the between-class variance. The threshold obtained by this strategy is equal to the average of the mean levels of two classes partitioned by this threshold. However, the threshold achieved with maximizing the betweenclass variance divides the mixture into two parts, one belongs to the land part and the other belongs to water. It cannot solve the uncertainty mentioned above and provide the desired threshold. 


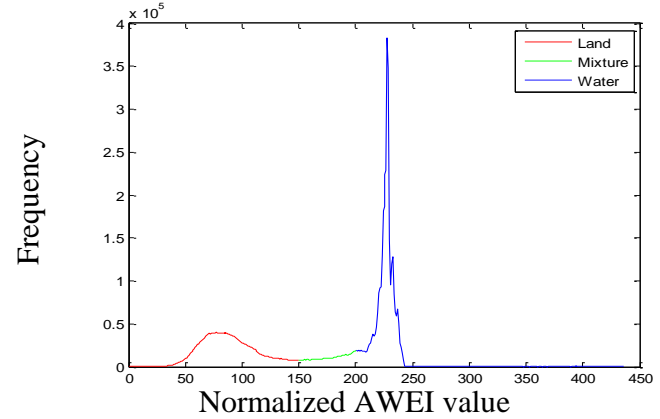

b

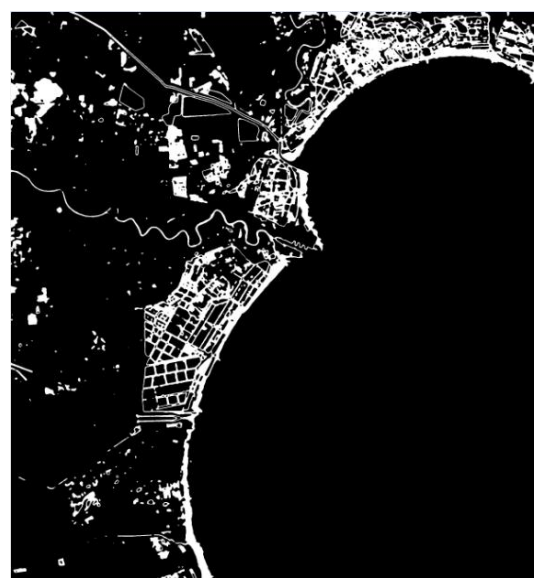

Figure 1. a) The histogram of normalized AWEI with the image in 1986 . b) The mixture part with white part.

In UCCD method, the improved strategy is used. With the normalized AWEI, the results of between-class variance of water and land is obtained. Then, the inflection point between mixture and pure water is determined as the optimal threshold for water-land separation by discrete first derivative computation of the between-class variance. Finally, after the
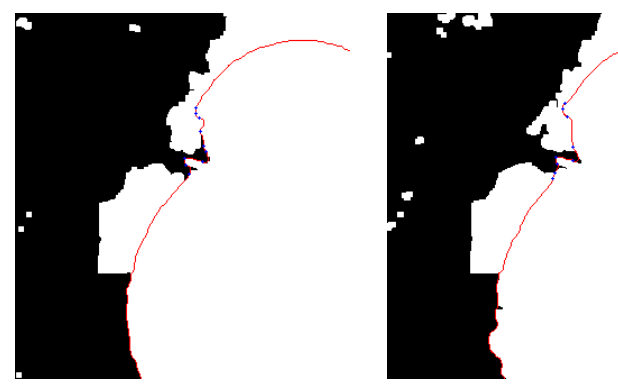

1986

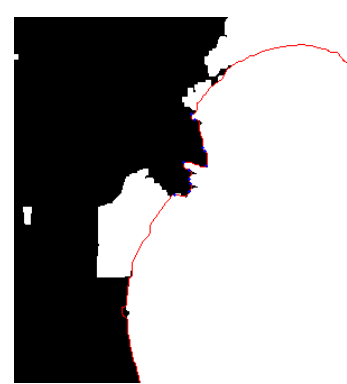

2006
1991

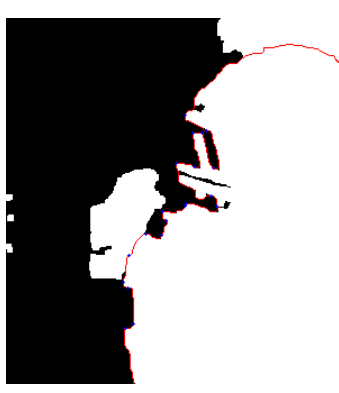

2011

post processing, the coastline position can be detected with boundary tracking. This threshold can effectively separate mixture to land part and detect accurate and reasonable coastline position (waterline).

\subsection{Subtype coastline determination with multiscale feature}

Based on the accurate coastline position, the MFBSCD can effectively extract the globe and local features of coastline with considering scale-invariant of features. It not only can guarantee obtaining wanted features, but also can transform the data from high scale to the low with sharply reducing the redundancy of the data and promoting the computational efficiency. The detailed steps can be described as follows: 1) the features of the coastline are displayed under different scale, the selection of optimal scale for scale-invariant features extraction is conducted, 2) the scale-invariant features extraction under the selected scale, 3) the subtype coastlines are extracted by multilayered classification and cross-validation.

With the study area chosen in this paper, each subtype coastline has its own typical characteristics which are mainly reflected in geometry and spatial structure. However, in the original scale, the features of coastline are much complex, and it is difficult to obtained effective features. Therefore, based on the theory of scale-invariant, the geometrical and spatial structure features of the coastline can be described and displayed under different scale through the Gaussian pyramid establishment. Take the coastline from the image in 1986 for example, total 5 layers are established ( 0 to 4 th), and the width and height of each layer are respectively 2608x1398, 1304x1199, 652x599, 326x299 and $163 \times 149$. The geometry of the coastline is described by corners extracted by Harris algorithm, and spatial structure of it is represented by separated result of water and land. With the scale down, the quantity of reserved features are reduced. When under the fourth layer, the features in geometry and spatial structure are damaged seriously. The quantity of corner of each layer is respectively $580,264,116,57$ and 23 . The relative minimum reduction rate of corner amount is $50.86 \%$ between

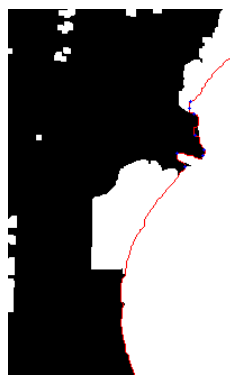

1996

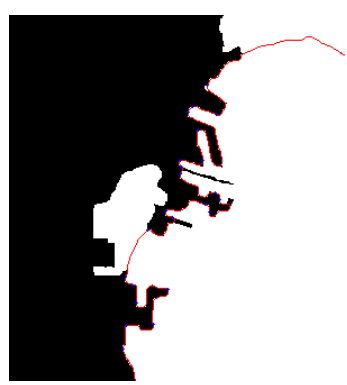

2016

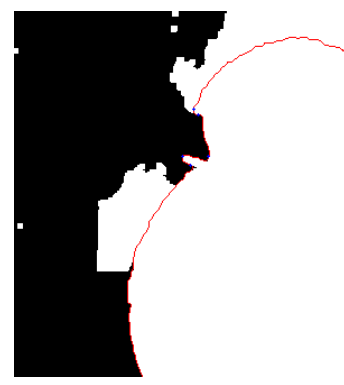

2001
- Corners

_ Coastline position

Water part

Land part

Figure 2. Extracted scale-invariant features of the coastlines in the selected scale. 
the third and fourth layer, and relative maximum of the reduction rate is $59.64 \%$ between the third to the fourth layer. In the third layer, the features of coastline are reserved better with reducing the data redundancy as much as possible, and some ineffective features can be eliminated.

With the global geometrical and spatial structure features of coastline, the local effective features for the determination of the each subtype coastline should be obtained under the scale selected. The MFBSCD method can effectively extract the strong geometrical features with high complexity and tortuosity by selecting the corners which is chosen to describe the geometry of coastline for determining the port coastline. The specific steps are as follows. Firstly, corners of the whole coastline are extracted by Harris algorithm, and initial appropriate number of corners are selected with the threshold of the energy function values. Then, for the each initial selected corner, the absolute slope difference of the connected straight line between it and the two adjacent corners are computed. The corner with higher absolute slope difference value are reserved. Next, based on the Euclidean distance between two adjacent corners, the remaining corners are clustered into several groups. Finally, in each group, the ratio value of coastline length and the Euclidean distance between the two corners with the furthest distance is computed, and the corners in the group with higher ratio value are reserved. The final selected corners are all located at the section with high complexity and tortuosity in geometry (Figure 2). At the same time, the spatial structure features which is another important aspect should be considered The spatial structure of coastline mainly manifest in the objects located in neighboring areas of the coastline. They are also indicators that directly related to the subtype of coastline. In the study area in this paper, there are salt pans in the coastal regions, and water characteristic in the neighboring area is strong feature. The MFBCE method extracts this feature by computing the mean value of each coastline position in the neighboring area based on the separated results of water and land, and the mean value is represented by M_A. The size of neighboring window

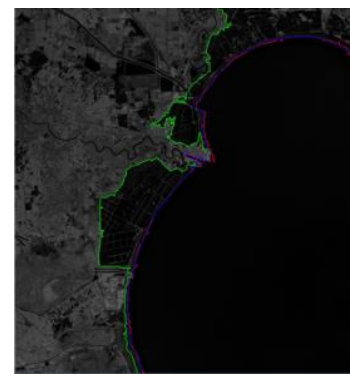

1986

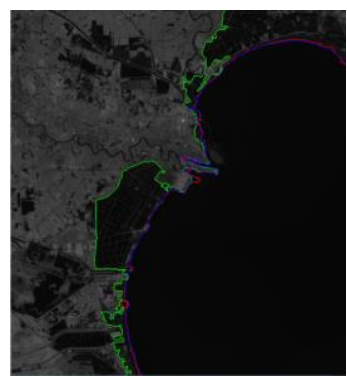

2006

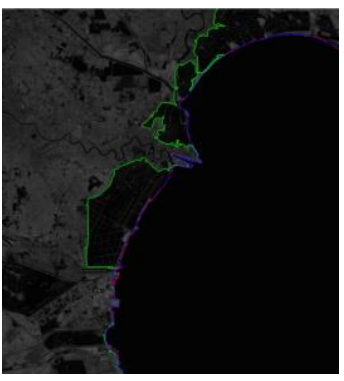

1991

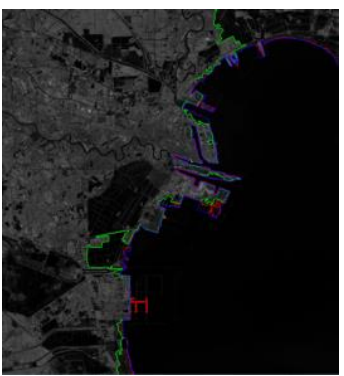

2011

is $3 \times 3$ with nine pixels contained (Figure 2). Choosing waterland separated result to replace the normalized AWEI result makes the extraction of spatial structure feature have more robustness for the size of neighboring window.

Based on multilayered classification and cross-validation, the port coastline is determined at the region with corners and low M_A. The range of saltpan coastline is obtained with high M_A The region with low M_A and no selected corners is the range of the construction coastline. Based on the ratios of scale establishment, the ranges of the subtypes in the original scale of the images are final achieved.

\section{RESULT AND CONCLUSION}

Based on the manually delineated coastlines, the comparisons between the coastlines position detected by MFBSCD method and OTSU method were conducted, and the accuracy assessments were also conducted on all the determined subtype coastlines. The two statistical measures including misclassification error (ME) and Line Matching (LM) are chosen.

As shown in Figure 3, the mean ME and mean LM of the coastline obtained by the MFBSCD method are respectively 0.0012 and $0.0245 \mathrm{~km}$, and for OTSU method, they are respectively 0.1810 and $3.5794 \mathrm{~km}$. The coastline position detected by OTSU method deviates the actual coastline position seriously. Meanwhile, in Figure 4, compared with the coastline delineated manually, the mean LM of the port, saltpan and construction coastline is respectively $0.0545 \mathrm{~km}, 0.0641 \mathrm{~km}$ and $0.1003 \mathrm{~km}$, and the mean ME is respectively $0.0388,0.0243$ and 0.0561 .

The results demonstrate that this method can successfully detect the accurate coastline position and achieve automated determination of subtype coastline.

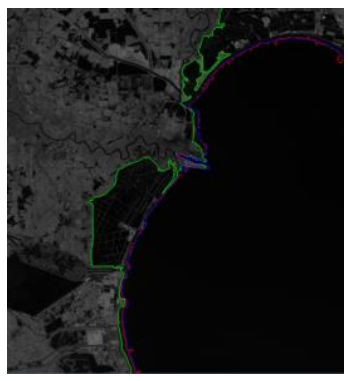

1996

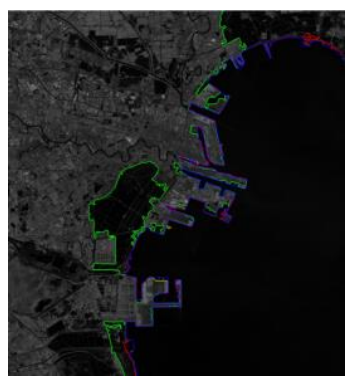

2016

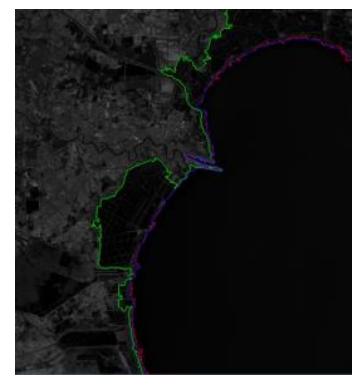

2001

Our method

Otsu method

Manually

Figure 3 . The coastline position detected by different method. 


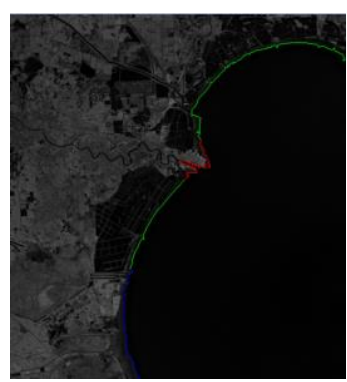

1986

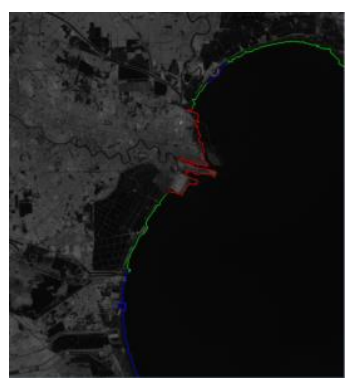

2006

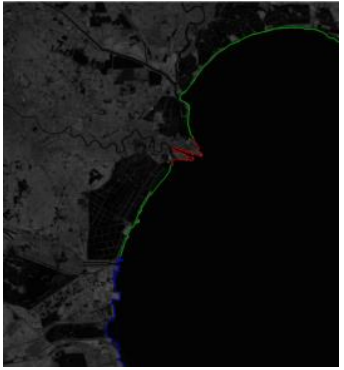

1991

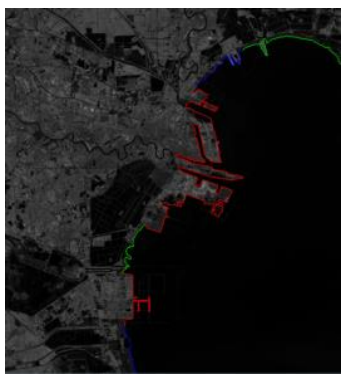

2011

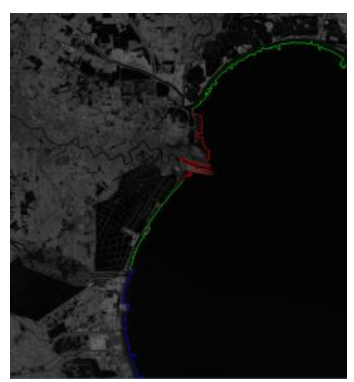

1996

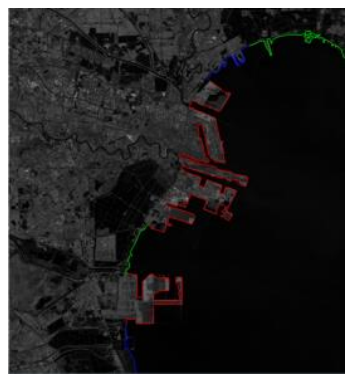

2016

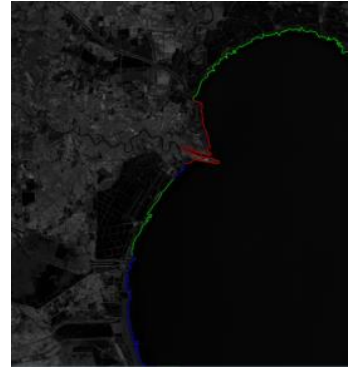

2001

Port coastline

Saltpan coastline

Construction coastline

Figure 4. Automated subtype coastline determination by MFBSCD method from Landsat imagery in Tianjin, China.

\section{ACKNOWLEDGEMENTS}

This work was supported by Natural Science Fund of Hubei Province (No.2016CFB690) and The Fund of Key Laboratory of Technology for Safeguarding of Maritime Rights and Interests and Application (No.SCS1610).

\section{REFERENCES}

Dewan, A.M., Yamaguchi, Y., 2008. Effect of land cover changes on flooding: Example from greater Dhaka of Bangladesh. Int. J. Geoinform. 4, pp. 11-19.

Du, Z., Li, W., Zhou, D., 2014. Analysis of Landsat-8 OLI imagery for land surface water mapping. Remote Sens. Lett. 5, pp. $672-681$

Ding, X.W., Li, X.F., 2014. Shoreline movement monitoring based on SAR images in Shanghai, China. Int. J. Remote Sens. 35, pp. 3994-4008.

Dewi, R.S., Bijker, W., Stein, A., 2016. Fuzzy classification for shoreline change monitoring in a part of the northern coastal area of java, Indonesia. Remote Sens. 8, pp. 190.

Duru, U., 2017. Shoreline change assessment using multitemporal satellite images: a case study of Lake Sapanca, NW Turkey. Environmental Monitoring and Assessment. 8.

Gens, R., 2010. Remote sensing of coastlines: Detection, extraction and monitoring. Int. J. Remote Sens. 31, pp. 18191836.
Ghosh, M.K., Kumar, L., Roy, C., 2015. Monitoring the coastline change of Hatiya Island in Bangladesh using remote sensing techniques. ISPRS J. Photogramm. Remote Sens. 101, pp. 137-144.

Gu, Z., Jia, P.H., Li, G.C., 2016. Research on A Technique of Extracting Coastline Shoreline Informationextraction Using The Canny Edge Detection Operator for Two Coastal Lagoons in Lingshui, Hainan Province. Quaternary Sciences. 1, pp. 113120.

Klemas, V., 2011. Beach profiling and LiDAR bathymetry: An overview with case studies. J. Coast. Res. 27, pp. 1019-1028.

Li, W., Gong, P., 2016. Continuous monitoring of coastline dynamics in western Florida with a 30-year time series of Landsat imagery. Remote Sens. Environ. 179, pp. 196-209.

Liu, X.Y., Gao, Z.Q., Ning, J.C., 2016. An Improved Method for Mapping Tidal Flats Based on Remote Sensing Waterlines: A Case Study in the Bohai Rim, China. IEEE Journal of Selected Topics in Applied Earth Observations and Remote Sensing. 11, pp. 5123-5129.

Muslim, A.M., Foody, G.M., Atkinson, P.M., 2006. Localized soft classification for super-resolution mapping of the shoreline. Int. J. Remote Sens. 27, pp. 2271-2285.

Pardo-Pascual, J.E., Almonacid-Caballer, J., Ruiz, L.A., 2012. Automatic extraction of shorelines from Landsat TM and ETM+ multi-temporal images with subpixel precision. Remote Sensing of Environment. 123, pp. 1-11. 
Tamassoki, E., Amiri, H., Soleymani, Z., 2014. Monitoring of shoreline changes using remote sensing (case study: Coastal city of Bandar Abbas). In Proceedings of the IOP Conference Series: Earth \& Environmental Science, Kuala Lumpur, Malaysia, 2223 April, pp. 12023.

Vaidya, A.M., Kori, S.K., Kudale, M.D., 2015. Shoreline response to coastal structures. Aquat. Procedia. 4, pp. 333-340.

Wang, C.Y., Zhang, J., Ma, Y., 2010. Coastline interpretation from multispectral remote sensing images using an association rule algorithm. Int. J. Remote Sens. 31, pp. 6409-6423.

Wang, X., Liu, Y.L., Ling, F., 2017. Spatio-Temporal Change Detection of Ningbo Coastline Using Landsat Time-Series Images during 1976-2015. ISPRS Int. J. Geo-Inf. 6, pp. 68.

Wang, C.Y., Wang, Z.R., Chu, J.L., 2017. Coastline extraction $f$ rom high-resolution image based on decision tree and density $\mathrm{cl}$ ustering algorithms. Marine Environmental Science. 36, pp. 590 -595 .

Xu, X.G., Li, X., Chen, M.C., 2016. Land-ocean-human interactions in intensively developing coastal zone: Demonstration of case studies. Ocean \& Coastal Management. 133, pp. 28-36.

Yang, C.X., Wang, J.Y., Huang, W.Q., 2017. An Improved Binary Image Method of Extracting Shoreline Based on LiDAR Data. Geomatics and Information Science of Wuhan University. 7, pp. 897-903

Zhang, T., Yang, X.M., Hu, S.S., 2013. Extraction of Coastline in Aquaculture Coast from Multispectral Remote Sensing Images: Object-Based Region Growing Integrating Edge Detection. Remote Sens. 5, pp. 4470-4487.

Zhan, Y.T., Zhu, L., Sun, Y.H., 2017. Automatic extraction of coastline via spectral angle-distance similarity growth model. Journal of Remote Sensing. 3, pp. 458-469.

Zhou, Q., Qu, C.W., Li, J.W., 2017. Extracting small harbor areas in large-scene SAR images. Journal of Image and Graphics. 22, pp. 1128-1134. 